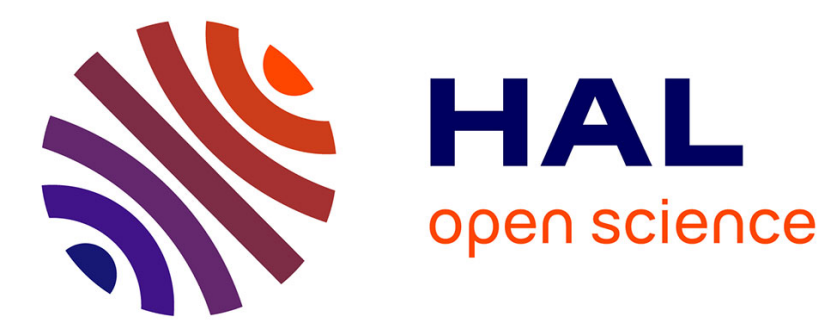

\title{
Effect of Slag Impregnation on Thermal Degradations in Refractories
}

\author{
Eric Blond, Nicolas Schmitt, François Hild, Philippe Blumenfeld, Jacques \\ Poirier
}

\section{- To cite this version:}

Eric Blond, Nicolas Schmitt, François Hild, Philippe Blumenfeld, Jacques Poirier. Effect of Slag Impregnation on Thermal Degradations in Refractories. Journal of the American Ceramic Society, 2007, 90 (1), pp.154-162. 10.1111/j.1551-2916.2006.01348.x . hal-00299449

HAL Id: hal-00299449

https://hal.science/hal-00299449

Submitted on 22 Mar 2018

HAL is a multi-disciplinary open access archive for the deposit and dissemination of scientific research documents, whether they are published or not. The documents may come from teaching and research institutions in France or abroad, or from public or private research centers.
L'archive ouverte pluridisciplinaire HAL, est destinée au dépôt et à la diffusion de documents scientifiques de niveau recherche, publiés ou non, émanant des établissements d'enseignement et de recherche français ou étrangers, des laboratoires publics ou privés. 


\title{
Effect of Slag Impregnation on Thermal Degradations in Refractories
}

\author{
Eric Blond, Nicolas Schmitt, and François Hild \\ LMT-Cachan (ENS de Cachan/CNRS-UMR 8535/Université Paris 6), F-94235 Cachan Cedex, France
}

\author{
Philippe Blumenfeld \\ Arcelor Research, Voie Romaine, F-57283 Maizières-les-Metz, France
}

\author{
Jacques Poirier \\ CRMHT-CNRS, F-45071 Orléans Cedex, France
}

\begin{abstract}
Degradation in bauxite refractory linings used in steel plants is localized in the slag-impregnated zone of refractories. It is proposed to estimate the contribution of the pressure of the impregnated liquid on the degradation of refractories. The study of the gradient of chemical composition allows for the evaluation of the liquid content, the oxide content in the liquid phase, and the "slag" viscosity. The thermomechanical analysis of a porous refractory impregnated by slag enables us to propose analytical expressions for a rapid estimate of the maximum "slag pressure" and its location as well as the level of thermoelastic stresses.
\end{abstract}

slag but also on the rotations of the ladle: the more the ladle is used, the less the wear observed!

Numerous studies have examined the effects of contact between the refractory and slag or other liquids to better understand the role played by the chemical composition, the mineralogy, and the microstructure in the corrosion mechanism. ${ }^{1}$ The most common thermomechanical degradations of refractory working linings during the steel-making process are cracking and spalling by thermal shock. The material properties and structural effects often explain these degradations. ${ }^{4-8}$ For about 2 decades, many studies have focused on the non-linear mechanical behavior of the material; however, they are hardly ever used in numerical simulations of the whole lining.

In the next section, the reasoning followed to build a multiphysics coupling framework for the modeling of working lining degradations is shown. Then, in the following sections, two phenomena resulting from the coupling effects are discussed in the case of bauxite refractory linings:

(1) the change of physical properties (liquid content, oxide content, and viscosity) due to the coupling between phase changes and impregnation, and

(2) the fluctuation of the "slag" pressure and the stresses due to the interaction between thermomechanics and slag impregnation.

\section{A Multi-Physical Couplings Analysis}

To understand the degradation process in refractories, the usual approach consists in listing and classifying the loading parameters. In the specific case of refractories used in steelmaking plants, working linings are subjected to three major types of load histories:

(1) Thermal loadings. The refractory lining is subjected to thermal gradients and to cyclic thermal loadings due to the steelmaking process, namely, high heating rate during the filling stage of the vessel, prescribed surface temperature during the refining process (inner ladle), high cooling rate during the first minutes of the emptying stage, and low cooling rate during dwell and maintenance stages. This complex thermal history generates thermal strain and/or stress and also possible phase changes. Temperature variations also influence the corrosion rate.

(2) Mechanical loadings. Gravity forces have insignificant effects for stiff and resistant materials used in small structures but the effects are severe when the materials are soft and weak, and the structures are tall and heavy. The turbulence of the metal and slag bath during the refining process generates dynamic loads on the refractory surface causing erosion. On the microscopic scale, capillarity forces are also considered as loading. The latter is responsible for the impregnation of the refractory by slag.

(3) Chemical loadings. These loads result from non-equilibrium between mineralogical phases, namely, the difference in the 
chemical potential of the different phases leads to chemical diffusion or reactions. Typical consequences are phase transformations, dissolution, and corrosion. . $^{1,9,10}$

This classification suggests a coupling diagram with three poles, namely, a heat transfer pole, a chemical pole, and a mechanical pole. However, this choice does not give a well-adapted framework to deal with the degradation of refractories. The review of possible causes of lining damage in industrial reactors, such as the formation of a "brittle layer" in a blast furnace ${ }^{11,12}$ or the degradation of the lining in a vacuum-oxygen decarburization (VOD) reactor, ${ }^{13}$ involves three major phenomena ${ }^{14}$ namely, thermomechanics, slag impregnation by capillarity, and phase changes. Impregnation is an important transfer vector for reactive products through the lining and it is impossible to describe it easily in this first coupling diagram. Moreover, the chemical pole is linked to impregnation because chemical reactions occurring in the impregnated zone of refractories depend on the nature of the liquid phases. Consequently, to develop predictive models for the degradation process, a framework based on multi-physical couplings between the poles thermomechanics, impregnation, and phase transformations is proposed.

Classical degradations observed in different metallurgical reactors at various stages of the steel making process are caused by mechanisms that are described in this framework:

(1) Temperature favors phase changes that produce bulk variations and stresses. Thermally activated phase changes also modify the thermomechanical behavior of the material.

(2) Capillary impregnation brings reagents, inducing thermally activated phase changes. Conversely, phase changes stop capillary impregnation by healing microcracks and closing pores.

(3) Impregnation is activated by capillary suction and made easier by mechanical load such as ferrostatic pressure. Furthermore, differential thermal expansion between pores and liquid oxides generates fluid mass flows. Last, the presence of liquid in pores may change the mechanical behavior of the whole lining.

From a conceptual point of view, if one were able to model all the mechanisms in the couplings diagram, one would be able to account for all possible causes of degradations in a single model. However, it is very difficult to model the degradation processes in their full complexity. Consequently, the decomposition of the whole problem into several simpler sub-problems is necessary to bypass this difficulty.

Let us consider refractory lining in contact with molten slag. The impregnation of refractory by slag depends on the wetting angle, the connected porosity, its tortuosity, and the reactivity of slag with the solid compounds. If impregnation occurs, the thickness of the lining is divided into two zones, namely an impregnated zone and an unimpregnated one with moving boundaries (Fig. 1). Near the surface in contact with slag, the impregnated zone is dissolved. Consequently, the surface moves inside the brick with a rate close to $1-2 \mathrm{~mm} / \mathrm{h}$ for bauxite refractories. But for the steel-refining process, the dissolution of refractories is not regular; it depends both on the temperature and composition of the liquid in contact (steel or slag). The kinetics of impregnation is controlled by heat transfer or fluid mass flow. For fast fluid mass flows, the surface separating the unimpregnated zone from the impregnated one corresponds to the isotherm of solidification for slag. The thickness of the impregnated layer is related to the thermal gradient. For slow fluid mass flow, the surface is limited by the fluid flow rate and the thickness is due to the competition between dissolution rate and fluid flow rate. In use, the impregnation of refractory with slag occurs quickly ${ }^{15,16}$ and after a transient regime where the depth of the impregnated zone grows, the thickness of this layer remains constant. Depending on temperature and slag viscosity, capillary penetration may stop before the slag reaches chemical equilibrium with the refractory. In such a case, the penetration is followed by a long hold time at a high constant temperature (with a gradient or not) so that chemical equilibrium between slag oxides and refractory oxides may be reached before cooling down. For high alumina/basic slag systems, during first contact, liquid oxides dissolve the solid phases of the refractory that are
Refractory

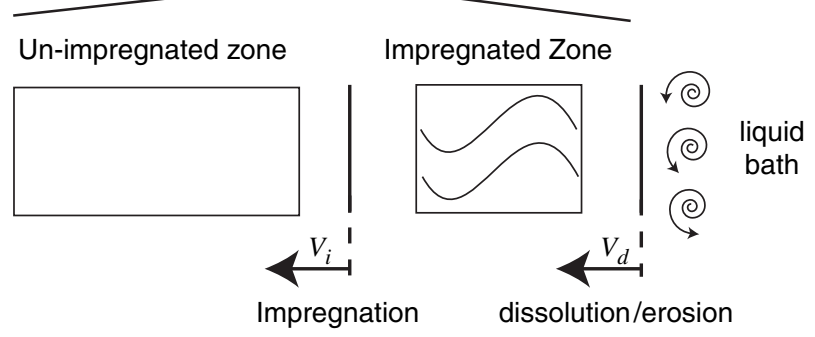

Fig. 1. Spatial decomposition of slag impregnation/corrosion mechanisms. An impregnated and an unimpregnated zone is depicted.

not in equilibrium with them and new phases are precipitated. Solid-liquid interface reactions are faster than chemical species bulk transfers; thus, an assumption of local chemical equilibrium is made..$^{9,10}$

The modeling of the coupling between impregnation and thermomechanics leads us to consider the slag-impregnated zone as a porous medium, for which the refractory is the skeleton, gases, and molten slag, which filled the porosity the fluid phases. The fluids influence the thermomechanical behavior of the whole medium. Furthermore, the liquid phase constitutes a chemical reagent source (or well) for the skeleton and vice versa. This complexity is modeled by considering two problems, one for each medium, coupled via their boundary conditions. It is possible to study separately the kinetics of each medium (skeleton/fluid) with the proposed coupling diagram. The complementary medium (fluid for example) prescribes mechanical boundary conditions (to the skeleton). Momentum balance and conservation laws link these two problems (skeleton/fluid).

For each case, it is important to identify the dominant interactions because they are not equally important. The previous general framework is used to identify the main factors controlling spalling of high-alumina-based working linings of steel ladles (Fig. 2).

\section{Impregnation-Phase Transformations}

\section{(1) Experimental Procedure}

The microstructure of a bauxite refractory used in steel ladles was characterized at room temperature (RT) using a scanning electron microscope (SEM, Hitachi S-510, Tokyo, Japan) and an energy-dispersive spectrometry (EDS) analyzer (Princeton Gamma-Tech XS14-H005, Princeton, NJ). Microanalyses were performed on small plates $(3 \mathrm{~mm} \times 4 \mathrm{~mm})$ taken at several depths from specimens parallel to the corroded face. Thermochemistry simulations with the thermodynamic software CEQSI $^{17}$ based on data collected by EDS were also carried out to estimate the likely state of oxides existing at a high temperature.

The analysis was first performed on a crucible corroded by slag at a temperature of $1600^{\circ} \mathrm{C}$ using a cup test. ${ }^{1}$ The thermal and chemical environments created in the crucible are not identical to those present in a steel plant, but this test enables one to

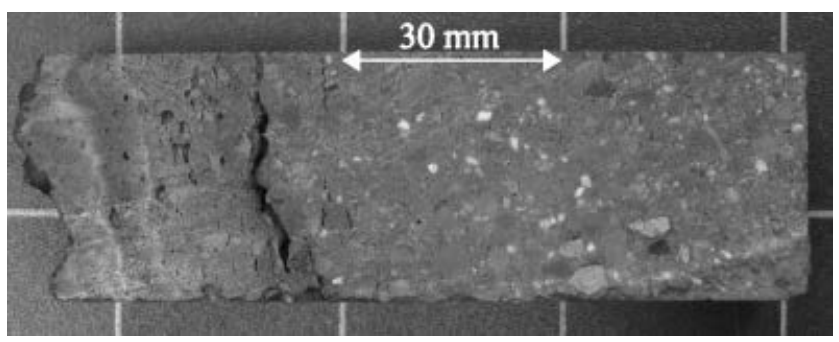

Fig. 2. Microstructure and degradation of a worn bauxite-based brick. A spalling zone located at $45 \mathrm{~mm}$ from the worn face is observed. 

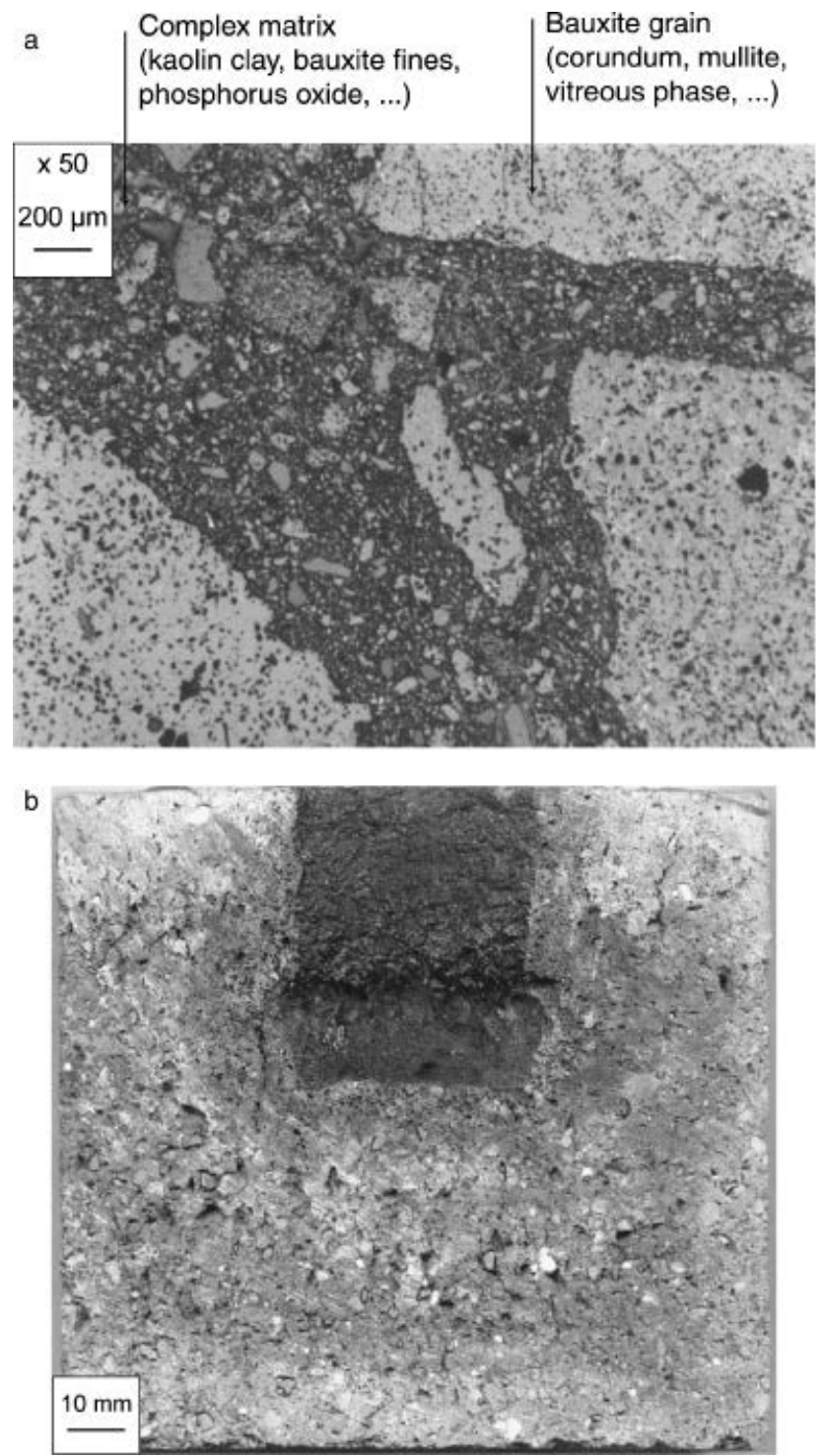

Fig. 3. (a) Microstructure of a bauxite-based refractory (before slag impregnation). (b) Crucible after impregnation $\left(5 \mathrm{~h}\right.$ at $1600^{\circ} \mathrm{C}$ in air).

identify markers for the different impregnated zones. The results guided us for the subsequent analysis of a worn sample taken from a steel ladle lining.

The commercial bauxite-based bricks studied herein are made of coarse aggregates of bauxite, with a bounding phase composed of kaolin clay, bauxite fines, and phosphorus pentoxide $\left(\mathrm{P}_{2} \mathrm{O}_{5}\right)$; see Fig. 3a. The microstructure of a grain of bauxite consists of fine corundum, mullite, aluminum titanate crystals, and vitreous phases. The average oxide content at RT is given (by the supplier) in Table I. The refractory is mainly made of alumina $\left(\mathrm{Al}_{2} \mathrm{O}_{3}\right)$ and silica $\left(\mathrm{SiO}_{2}\right)$. The material contains a low quantity of iron oxide $\left(\mathrm{Fe}_{2} \mathrm{O}_{3}\right)$ and titanium dioxide $\left(\mathrm{TiO}_{2}\right)$. At RT, the connected porosity varies from $18.5 \%$ to $24 \%$.

The crucible test was carried out on a brick with a square section of $110 \mathrm{~mm} \times 110 \mathrm{~mm}$ and a height of $100 \mathrm{~mm}$ in which a
$37 \mathrm{~mm}$ diameter hole is drilled (depth $=45 \mathrm{~mm}$ ). The hole was filled with synthetic slag. This pre-melted slag is composed of lime, alumina, iron, and iron oxides $\left(\mathrm{Fe}_{2} \mathrm{O}_{3}\right.$ and $\mathrm{FeO}$ at a high temperature) and is representative of a composition impregnating refractories in steel ladles (Table I). The temperatures of solidus $\left(1049^{\circ} \mathrm{C}\right)$ and liquidus $\left(1188^{\circ} \mathrm{C}\right)$ of this synthetic slag were estimated with the software CEQSI. ${ }^{17}$ After reaching the melting point, a basic slag with a lime/alumina weight ratio close to 1.2 and a total iron content of $30 \mathrm{wt} \%$ was obtained. The crucible was heated in air in an electric furnace at $5^{\circ} \mathrm{C} / \mathrm{min}$ and held for $5 \mathrm{~h}$ at $1600^{\circ} \mathrm{C}$ to avoid thermal cracks. The furnace was then switched off and naturally cooled to RT.

\section{(2) Results}

The crucible, cut perpendicular to the bottom face of the hole, is shown in Fig. 3b. The microstructure of the impregnated zone depends essentially on the depth from the interior. Let us analyze the refractory below the bottom of the hole. Along the first $10 \mathrm{~mm}$, the microstructure is homogeneous on a macroscopic scale, except the closed spherical porosity, which is probably due to degassing of slag. For depths varying between 15 and $30 \mathrm{~mm}$, the grains look like the original ones, but the texture of the bounding phase is different from the initial one. Beyond the 35$\mathrm{mm}$ depth, the initial microstructure is unchanged. A fine study of microstructure changes was performed by Quafssaoui. ${ }^{9}$ Four zones are distinguished: a remnant slag zone, a precipitation zone where calcium aluminates are formed, an impregnated zone, and an unimpregnated zone.

By averaging EDS analyses over the $4 \mathrm{~mm} \times 3 \mathrm{~mm}$ plates, the profiles of oxides formed with the chemical elements $\mathrm{Al}, \mathrm{Si}, \mathrm{Ca}$, $\mathrm{P}, \mathrm{Fe}$, and $\mathrm{Ti}$ were obtained with a relative error on the mass content less than $4 \%$. The analysis focused on the oxides characterizing the impregnation process, namely $\mathrm{P}_{2} \mathrm{O}_{5}$ and titanium dioxide $\left(\mathrm{TiO}_{2}\right)$ initially present in the bounding phase of the refractory, lime $(\mathrm{CaO})$, and iron oxide $\left(\mathrm{Fe}_{2} \mathrm{O}_{3}\right)$ contained in the initial composition of slag. They are regarded as indicators of the impregnation state, and their chemical content with depth is thus of particular interest.

As shown in Fig. 4, the profiles of oxides content contained initially in the slag mixture $\left(\mathrm{CaO}, \mathrm{Fe}_{2} \mathrm{O}_{3}\right)$ and those of oxides present in the refractory $\left(\mathrm{TiO}_{2}, \mathrm{P}_{2} \mathrm{O}_{5}\right)$ are different. The mass contents in $\mathrm{Fe}_{2} \mathrm{O}_{3}$ and $\mathrm{CaO}$ decrease quickly with depth down to asymptotic values reached at the 20-25-mm depth (Fig. 4a). The $\mathrm{TiO}_{2}$ and $\mathrm{P}_{2} \mathrm{O}_{5}$ mass contents first increase up to a maximum value, and then decrease down to their initial levels (Fig. 4b). One interpretation of these observations is that, along the first millimeters, the slag mixture dissolves the refractory phases that are not in equilibrium with it and new phases, $\mathrm{CaO}\left(\mathrm{Al}_{2} \mathrm{O}_{3}\right)_{n}$ and $\mathrm{Fe}_{2} \mathrm{O}_{3}$, are precipitated. ${ }^{18}$ When penetrating the brick, the liquid slag reacts with the solid oxide particles over the first millimeters. The composition of the liquid changes notably by addition of components of the dissolved grains $\left(\mathrm{Al}_{2} \mathrm{O}_{3}\right)$ and bounding phase $\left(\mathrm{P}_{2} \mathrm{O}_{5}, \mathrm{TiO}_{2}\right)$. As the liquid continues to seep into the refractory, the lime content becomes lower because the crucible hole initially filled with slag is emptied. This phenomenon is not observed in used bricks where the corroded face is regularly in contact with slag each time the vessel is emptied.

The liquid phase is considered as being locally in chemical equilibrium with the solid skeleton of the brick at each point because the kinetics of dissolution is faster than that of seeping. ${ }^{9,10}$ It is thus possible to estimate the content variation of the

Table I. Composition and Properties of Commercial Bauxite Refractory and Synthetic Slag

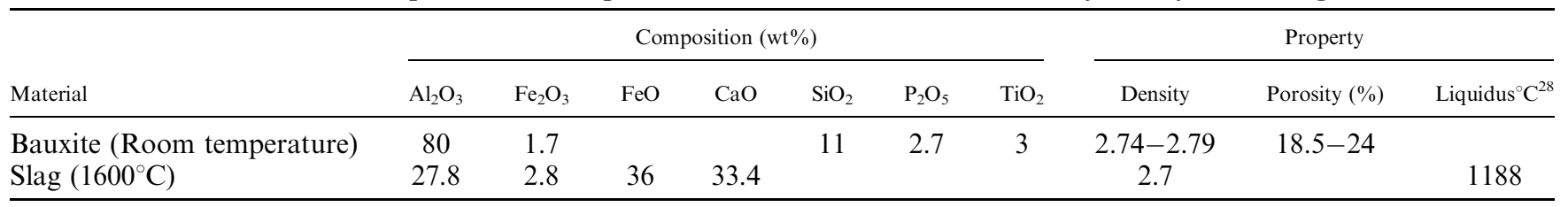



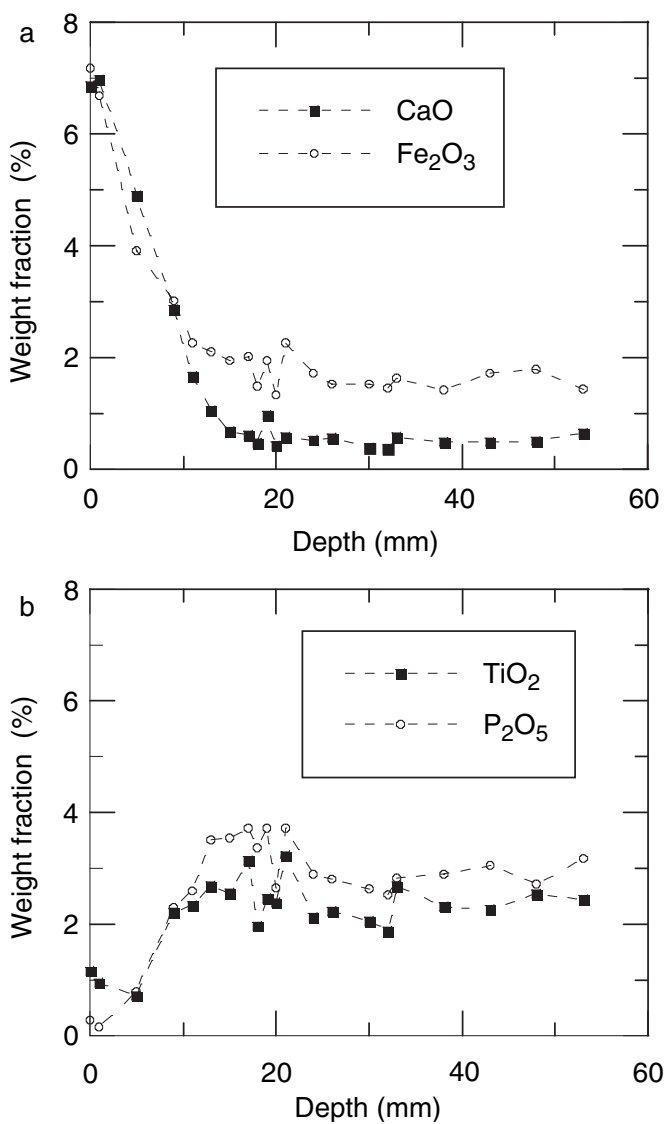

Fig. 4. $\mathrm{CaO}$ and $\mathrm{Fe}_{2} \mathrm{O}_{3}$ (a), $\mathrm{TiO}_{2}$ and $\mathrm{P}_{2} \mathrm{O}_{5}$ (b) profiles determined by energy-dispersive spectrometry (EDS) at room temperature for different depths in the tested crucible (Fig. 3(b)).

liquid phase present in the solid skeleton of the brick at a high temperature by thermo-chemistry computations. The software CEQSI ${ }^{17}$ was used to calculate the profile of the liquid content on the crucible at a high temperature. Phosphorus was not taken into account in this simulation; therefore, results have to be interpreted with caution, especially for the silica-bound phase where phosphorus is located. However, the phosphorous content is sufficiently small not to modify the phase distribution. The liquid content is underestimated as phosphorus has a tendency to decrease the melting point of silicate compounds. ${ }^{19}$ The $\mathrm{P}_{2} \mathrm{O}_{5}$ content at $\mathrm{RT}$ after impregnation and the estimated liquid content at $1600^{\circ} \mathrm{C}$ are shown in Fig. 5. The profiles reveal four microstructural zones for the $1600^{\circ} \mathrm{C}$ temperature. In the first 10 $\mathrm{mm}$, there is more than $40 \mathrm{wt} \%$ in liquid; it is a mushy/dissolved zone. From 10 to $23 \mathrm{~mm}$, the liquid content is close to the initial porosity. Considering the high $\mathrm{P}_{2} \mathrm{O}_{5}$ content, the liquid content is higher. Consequently, it is assumed that the liquid saturates the porosity. It constitutes the saturated impregnated zone. Beyond $23 \mathrm{~mm}$, an unsaturated impregnated zone exists, and farther away an unimpregnated zone; the boundary between zones is difficult to pinpoint accurately. These results show that the interstitial liquid is enriched with $\mathrm{P}_{2} \mathrm{O}_{5}$ along the first millimeters and deposits it farther away. The only possibility explaining the origin of the enriched content in $\mathrm{P}$ in the slag-impregnated zone is a convective transport of phosphorus caused by slag impregnation. Therefore, phosphorus present in the brick-bounding phase constitutes a natural chemical impregnation tracer. These observations and results were confirmed by Qafssaoui. ${ }^{9}$

If one assumes that phosphorus present in the brick-bounding phase is also leached in "real" refractory linings, phosphorus is a natural tracer to identify the liquid-impregnated zone of the worn brick, provided slag does not contain phosphorus (Figs. 2 and 6). Based on the previous considerations, the $\mathrm{P}_{2} \mathrm{O}_{5}$ profile confirms the visual observations, namely:

(1) a slag deposit, a depth of 0-5 $\mathrm{mm}$ with no $\mathrm{P}_{2} \mathrm{O}_{5}$,
(2) a boundary layer, a depth of 5-15 $\mathrm{mm}$ where the content in $\mathrm{P}_{2} \mathrm{O}_{5}$ increases,

(3) a large zone saturated in liquid at a high temperature, typically for depths ranging from 20 to $63 \mathrm{~mm}$, and

(4) an unimpregnated zone.

Figure 2 shows that the microcracks responsible for spalling are located $45 \mathrm{~mm}$ from the corroded face. Therefore, it is concluded that microcracks belong to the saturated and impregnated zone.

Although the hot face is often in contact with a high content of slag, the liquid phase does not impregnate a large zone of the brick. This is due to the fact that the temperature decreases with depth. When liquid seeps through the brick, it becomes more viscous with lower temperature, until it transforms into solid (Fig. 1). The depth of this transformation front varies with time because the temperature field changes.

These observations and the results of thermo-chemical simulations raise questions about the role played by the interstitial liquid in micro-crack initiation. This phenomenon is explained by modeling the coupling between impregnation and thermomechanics.

The previous results provide helpful information for modeling the porous-saturated medium. The in situ porosity existing at a high temperature is estimated from the liquid content. An estimate of the dynamic viscosity $v$ of the liquid phase, which is depending on both the chemical composition of the liquid and the temperature, was obtained using Urbain's model. ${ }^{20}$ It is important to note that the oxide content of the liquid considered for the estimation of the viscosity was not that of the original slag. Its composition is enriched in silica, and $\mathrm{P}_{2} \mathrm{O}_{5}$. For the impregnated crucible, for which the most reliable information concerning the phase content profile was collected, $v$ (estimated with Urbain's model) varies between 170 and $460 \mathrm{~Pa} . s$ at $1200^{\circ} \mathrm{C}$ and 0.6 and $1.2 \mathrm{~Pa}$.s at $1600^{\circ} \mathrm{C}$. Consequently, this property is more sensitive to temperature variations than composition changes.

\section{Impregnation-Thermomechanics}

In this section, the thermomechanical effects due to the presence of interstitial liquid during the steel-making process are shown. Material and process parameters and their influences are discussed. The impregnated zone is modeled as a porous medium filled with liquid, in which the refractory is the skeleton, and the molten "slag" is the saturating liquid.

Because of heat diffusion in a solid, the mechanical response of the porous medium to thermal loading is time dependent. For the porous medium, the liquid mass transfer in the porosity must also be considered and introduces a delayed mechanical response. Thermal fluctuations generate variations of liquid and porosity volumes, which induce fluid mass flow along the network of connected porosity due to pressure fluctuations.

The model proposed herein allows for a quick estimation of the "slag" pressure and the stresses induced within the refractory. It follows the same approach as the one proposed by Kingery ${ }^{6}$ and Hasselman ${ }^{7}$ for the derivation of thermal shock resistance criteria. This is performed by assuming that the mechanical behavior of the skeleton is linear elastic. The analysis becomes more difficult when the mechanical behavior is time dependent and/or the thermomechanical parameters depend on temperature. In these cases, numerical computations must be carried out.

\section{(1) Assumptions of the Model}

The model is written within the framework of the thermodynamics of continuous porous media. ${ }^{21}$ To analyze the main phenomena, some simplifying assumptions are made:

(1) The liquid and skeleton are assumed to be chemically inert. This assumption is reasonable in the saturated slag-impregnated zone after a short transient stage where both react together as the interstitial liquid in this zone quickly reaches chemical equilibrium with the skeleton. 


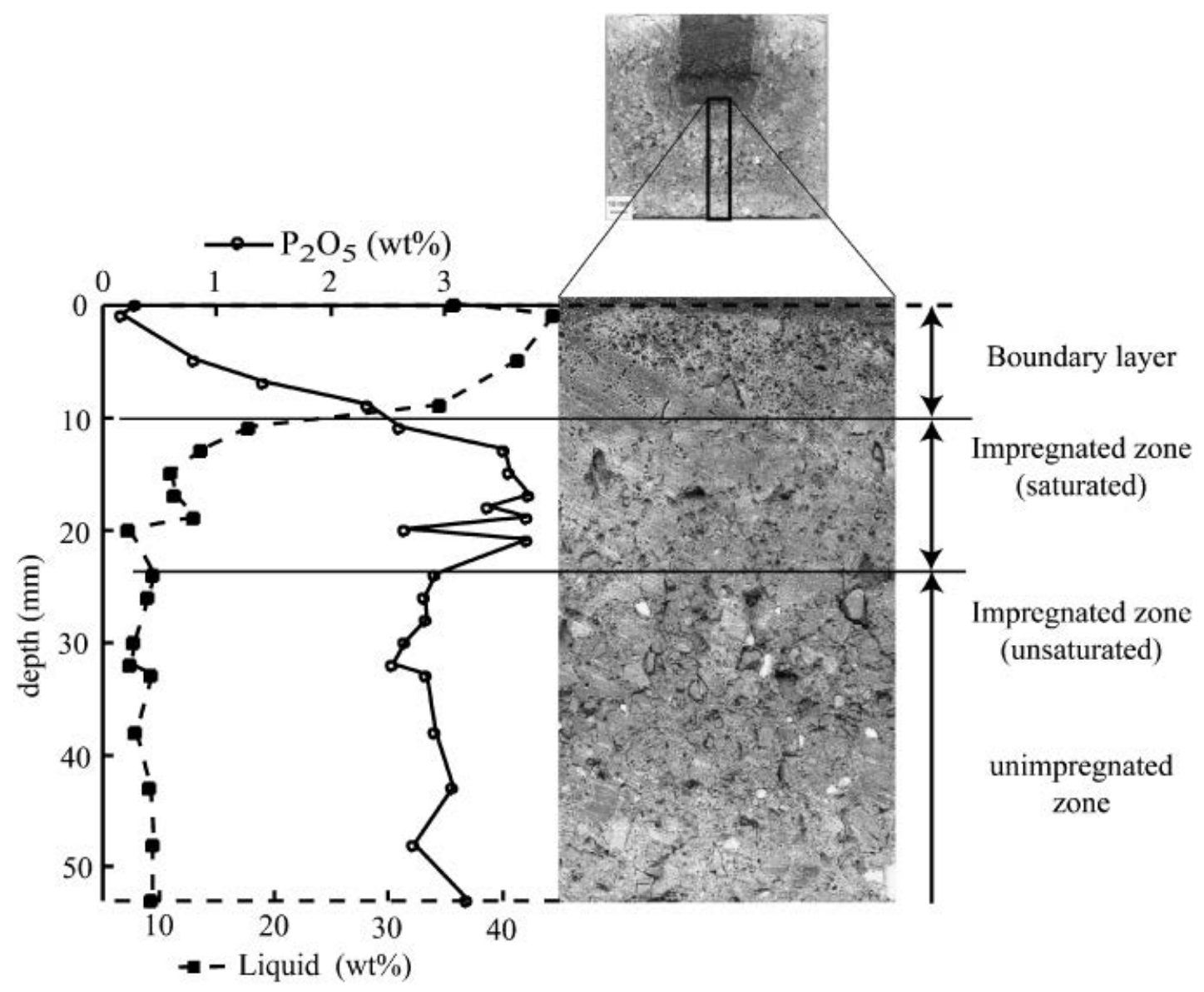

Fig. 5. Depiction of the four different zones in the tested crucible obtained from the profile of the impregnation tracer $\mathrm{P}_{2} \mathrm{O}_{5}$ determined at room temperature and the liquid weight fraction estimated at $1600^{\circ} \mathrm{C}$.

(2) The liquid phase is assumed to be a homogeneous phase throughout the impregnated zone. For example, the effects of the chemical composition of the liquid, the temperature, and the liquid-solid transformation occurring at temperatures close to $1200^{\circ} \mathrm{C}$ on viscosity are not accounted for. One practical consequence is that in the following derivations, the viscosity of the liquid phase does not depend on the depth and the temperature. In reality, the interstitial liquid may have its viscosity increase when it flows deeper into the lining. These assumptions are necessary in this first step to obtain qualitative results and main tendencies.

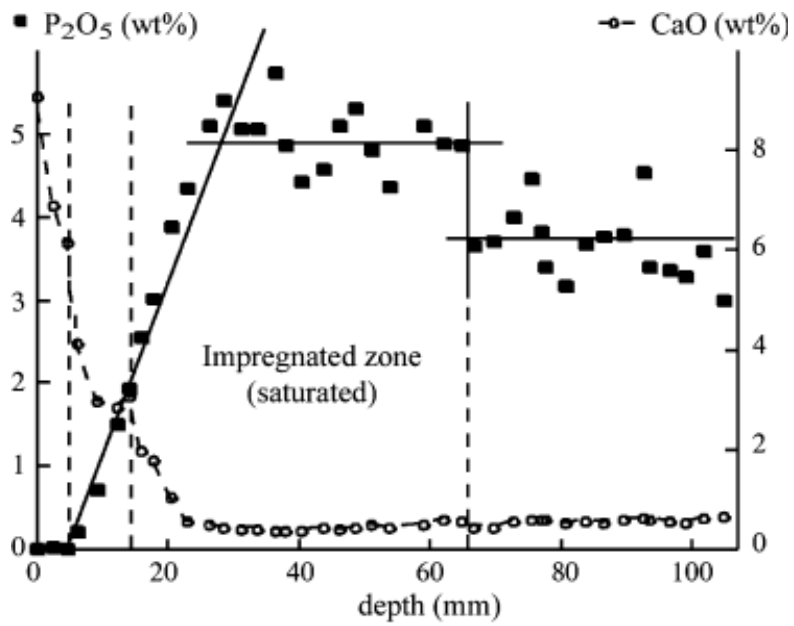

Fig. 6. $\mathrm{P}_{2} \mathrm{O}_{5}$ and $\mathrm{CaO}$ profiles determined at room temperature by energy-dispersive spectrometry (EDS) for different depths in a worn bauxite-based brick.
(3) Because of lack of information, porosity is also assumed to be constant with depth.

(4) The working lining is modeled as a one-dimensional half-space. This assumption is reasonable because the thickness of the bauxite brick is much greater than the wave length defined by the square root of the frequency divided by two times the diffusivity. ${ }^{22}$

(5) The skeleton has a thermoelastic behavior with temperature-independent properties considered for an average temperature of $1200^{\circ} \mathrm{C}$ (Young's modulus $E_{o}=5 \mathrm{GPa}$, Poisson's ratio $v_{\mathrm{O}}=0.2$, coefficient of thermal expansion $\alpha_{o}=6.9 \times$ $\left.10^{-6 \circ} \mathrm{C}^{-1}\right)$. Nonlinear features such as plasticity or creep are not accounted for.

(6) A harmonic surface temperature is prescribed on the free surface

$$
\theta(0, t)=\theta_{\max } \cos (\omega t+\phi)
$$

where $\theta_{\max }$ is the temperature amplitude and $\omega$ is the frequency of the thermal loading. Although this thermal loading does not describe the practical history during the process, it allows one to understand the role of a cyclic load on the behavior of the liquid present in the porous wall.

Under these assumptions, quasi-closed form solutions are obtained to estimate the maximum slag pressure and its location in the brick. As the stiffness of the porous material is significantly higher than in reality, the "slag pressure" is overestimated. Prevalent parameters are then identified.

\section{(2) Pressure and Stress Derivations}

To calculate the pressure $P(x, t)$, one has to solve two diffusion equations, namely, heat transfer (with Fourier's law) and liquid transfer (with Darcy's law), characterized by the thermal diffu- 
sivity $D_{\mathrm{T}}$ and the hydraulic conductivity $k$, respectively, and the mechanical equations that are partially coupled (see Appendix A). The problem reduces to a pressure diffusion equation, which is written for the one-dimensional setting $\mathrm{as}^{22}$

$$
\frac{\partial^{2} P(x, t)}{\partial x^{2}}-\frac{1}{D_{\mathrm{H}}} \frac{\partial P(x, t)}{\partial t}=\frac{\delta_{v} D_{\mathrm{T}}}{k} \frac{\partial^{2} \theta(x, t)}{\partial x^{2}}
$$

$\theta(x, t)$ is the temperature variation and $D_{\mathrm{H}}$ the hydraulic diffusivity, depending mainly on the hydraulic conductivity and modulus of compressibility of the skeleton and the fluid. The relative bulk expansion variation between the pores and the saturating liquid is characterized by the variable $\delta_{v}$, which includes the porosity, the relative thermal expansion, and the compressibility. For the considered application, $\delta_{v}$ is equal to $-9.5 \times 10^{-6} \mathrm{~K}^{-1}$.

The solution to Eq. (2) with the suitable boundary conditions is given by

$$
P(x, t)=-\frac{\mu^{2}}{1-\mu^{2}} \frac{P_{\mathrm{nd}}}{\theta_{\max }}[\theta(x, t)-\theta(\mu x, t)]
$$

where

$$
\theta(x, t)=\theta_{\max } \mathrm{e}^{-x / L_{\mathrm{c}}} \cos \left(\omega t-\frac{x}{L_{\mathrm{c}}}+\phi\right)
$$

is the temperature field, solution of the thermal diffusion equation with the suitable boundary conditions.

The diffusivity ratio $D_{\mathrm{r}}$, the square root of the inverse diffusivity ratio $\mu$, and the characteristic length $L_{\mathrm{c}}$ are defined by

$$
D_{\mathrm{r}}=\frac{D_{\mathrm{H}}}{D_{\mathrm{T}}}, \quad \mu=\sqrt{\frac{D_{\mathrm{T}}}{D_{\mathrm{H}}}}, \quad L_{\mathrm{c}}=\sqrt{\frac{2 D_{\mathrm{T}}}{\omega}}
$$

$P_{\text {nd }}$ is the undrained pressure, i.e. the "slag pressure" developed in a closed pore totally filled by liquid, subjected to a temperature variation of amplitude $\theta_{\max }$

$$
P_{\mathrm{nd}}=-\delta_{v}\left(\frac{1}{M}+\frac{b^{2}}{3 a K_{\mathrm{o}}}\right)^{-1} \theta_{\max }
$$

The pressure peak reaches a maximum value $P_{\max }$ during heating at the location $x_{\mathrm{cr}}$. This stage is critical to predict onset of damage caused by slag interstitial pressure. Conversely, upon cooling, the pressure reaches a negative minimum value. This may cause either slag suction, void nucleation, or both. In the particular case of a half-space submitted to a harmonic thermal loading with a frequency $\omega$, the critical dimensionless abscissa $X_{\mathrm{cr}}$, defined by the ratio of the real critical length $x_{\mathrm{cr}}$ to the characteristic length $L_{\mathrm{c}}\left(X_{\mathrm{cr}}=x_{\mathrm{cr}} / L_{\mathrm{c}}\right)$, is the solution to the following equation depending only on the parameter $\mu^{22}$

$$
\begin{aligned}
\mathrm{e}^{-(1-\mu) X_{\text {cr }}}+\mu \mathrm{e}^{(1-\mu) X_{\text {cr }}}= & (1-\mu) \cos \left[(1-\mu) X_{\text {cr }}\right] \\
& +(1-\mu) \sin \left[(1-\mu) X_{\text {cr }}\right]
\end{aligned}
$$

The location of the maximum pressure $P_{\max }$ depends on the material parameter $\mu$ and the thermal loading frequency $\omega$. It is worth noting that, for a monotonic load history, the pressure peak is not located at a given depth but propagates deep into the lining bulk with decreasing intensity. The characteristic length increases with time and is defined by ${ }^{23}$

$$
L_{\mathrm{c}}=\sqrt{2 D_{\mathrm{T}} t}
$$

Consequently, the location of maximum pressure mainly depends on the type of thermal loading, namely cyclic or monotonic. In the present case, the harmonic history is more relevant for the understanding of the degradation of refractory linings.

For a harmonic thermal loading, $L_{\mathrm{c}}$ (Eq. (5)) gives an estimate of the depth affected by the interstitial pressure fluctuation. Therefore, the depth affected by pressure fluctuation is thinner for faster ladle rotations than for slower ones. In practice, two cases of refining process limit the domain of the study, namely, a $130 \mathrm{~min}$ heating/70 $\mathrm{min}$ cooling for the faster ladle rotation and a $300 \mathrm{~min}$ heating/250 min cooling for the slower one. They correspond, as a first-order approximation, to a frequency $\omega$ of $5.24 \times 10^{-4}$ and $1.90 \times 10^{-4} \mathrm{rad} / \mathrm{s}$, respectively. Consequently, the characteristic length $L_{\mathrm{c}}$ varies between 50 and $84 \mathrm{~mm}$ for a thermal diffusivity of $D_{\mathrm{T}}=6.6 \times 10^{-7} \mathrm{~m}^{2} / \mathrm{s}$, which is typical of such refractories. ${ }^{14}$ One may note that the location of the maximum pressure moves several millimeters away from the hot face when the refining process period is longer.

Furthermore, the maximum pressure is proportional to the undrained pressure, itself proportional to the thermal amplitude ${ }^{22}$

$$
\begin{aligned}
\frac{P_{\max }}{P_{\text {nd }}}= & \left|\frac{\mu^{2}}{1-\mu^{2}}\right| e^{(1-\mu) X_{\text {cr }}} \\
& \times \sqrt{1+\mathrm{e}^{-2(1-\mu) X_{\mathrm{cr}}}-2 \mathrm{e}^{-(1-\mu) X_{\mathrm{cr}}} \cos \left[(1-\mu) X_{\mathrm{cr}}\right]}
\end{aligned}
$$

Using the usual equation of thermoelasticity of porous-saturated media, ${ }^{21}$ the knowledge of the slag pressure state allows one to compute the stress state in the half-space. The normal stress perpendicular to the free surface $\sigma_{1}$ reads

$$
\sigma_{1}(x, t)=0
$$

The normal stresses, $\sigma_{2}$ and $\sigma_{3}$, parallel to the free surface are identical

$$
\begin{aligned}
\sigma_{2}(x, t) & =\sigma_{3}(x, t) \\
& =\left(\frac{-2 \mu_{L}}{\lambda_{L o}+2 \mu_{L}}\right)\left[b P(x, t)+3 \alpha_{\mathrm{o}} K_{\mathrm{o}} \theta(x, t)\right]
\end{aligned}
$$

where $b$ is Biot's coefficient (very close to unity), $\lambda_{L o}$ and $\mu_{L}$ are Lamé's coefficients of the skeleton.

The effective normal stresses applied to the skeleton $\sigma_{i}^{\text {eff }}$ are given by

$$
\sigma_{i}^{\mathrm{eff}}(x, t)=\sigma_{i}(x, t)+b P(x, t) \quad i=\{1,2,3\}
$$

Equation (10) combined with Eq. (12) shows that the effective normal stress $\sigma_{1}^{\text {eff }}$ is proportional to the slag pressure. Consequently, its maximum value is reached at the same location and the same time as the slag pressure. When the slag pressure is positive, the skeleton is subjected to a tensile stress that is responsible for the opening of cracks parallel to the free surface.

By combining Eqs. (11) and (12), the following expression is obtained for the effective normal stresses $\sigma_{2}^{\text {eff }}$ and $\sigma_{3}^{\text {eff }}$

$$
\begin{aligned}
\sigma_{2}^{\mathrm{eff}}(x, t) & =\sigma_{3}^{\mathrm{eff}}(x, t) \\
& =\left(\frac{1}{1-v_{\mathrm{o}}}\right)\left[v_{\mathrm{o}} b P(x, t)-\alpha_{\mathrm{o}} E_{\mathrm{o}} \theta(x, t)\right]
\end{aligned}
$$

Contrary to the previous results (Eq. (12)), Eq. (13) shows that the effective normal stress $\left(\sigma_{2}^{\text {eff }}\right.$ or $\left.\sigma_{3}^{\text {eff }}\right)$ is a combination of two parts:

(1) a stress induced by the slag pressure (with a lower magnitude than $\sigma_{1}^{\text {eff }}$ because the strains are constrained in the plane of the wall), and

(2) a usual thermal stress induced by the thermal expansion of the skeleton. 
The magnitude of each part depends on the diffusivity ratio. These parts are either added or subtracted depending on the phase difference between the functions $P(x, t)$ and $\theta(x, t)$.

\section{(3) Discussion}

In steel-refining processes, the maximum temperature is prescribed whereas the minimum temperature depends mainly on the ladle operating cycle. Thus, the risk of failure is less likely when the ladle rotation is fast because the magnitude of the temperature variation in the lining is lower. In practice, if the minimum temperature becomes too low, burners are used to maintain a minimum temperature inside the vessel. Thermal computations with a finite element code have shown that the difference between the minimum temperatures for two limiting cases during the refining process does not exceed $100^{\circ} \mathrm{C}^{24}$ The usual temperature variation is approximately $\theta_{\max }=225^{\circ} \mathrm{C}$. In addition, considering the properties of refractories and slag at $1200^{\circ} \mathrm{C}$, the undrained pressure $P_{\text {nd }}$ (Eq. (6)) is approximately equal to $0.053 \theta_{\max }$. It reaches nearly $12 \mathrm{MPa}$ when $\theta_{\max }=225^{\circ} \mathrm{C}$. Equations (7) and (9) show that the dimensionless maximum pressure and its location only depend on the diffusivity ratio; and so does the effective normal stress $\sigma_{1}^{\text {eff }}$ (because $b$ is close to 1 ). It is possible to built master curves to define the level of crack risk and its location as a function of the diffusivity ratio. ${ }^{22}$

The thermal diffusivity is nearly constant for a wide range of refractories because its variation with composition is low, typically $\pm 2 \times 10^{-7} \mathrm{~m}^{2} / \mathrm{s}$. Yet, a high scatter of the hydraulic diffusivity $D_{\mathrm{H}}$ exists, as $D_{\mathrm{H}}$ is proportional to the hydraulic conductivity $k$, which depends on the morphology of the network of voids (porosity content, connectivity, tortuosity ${ }^{25}$ ), itself depending on the state of mineralogical phases, Poisson's ratio, and relative compressibility. This morphology and the mechanical parameters are expected to be different at high temperatures and at RT, where $k$ is usually identified by permeability measurements. The parameter $k$ is also proportional to the inverse of the dynamic viscosity $v$ of the liquid, which depends on temperature and its chemical composition. It is assumed that $D_{\mathrm{H}}$ ranges from $10^{-11}$ to $10^{-5} \mathrm{~m}^{2} / \mathrm{s}$. Consequently, the diffusivity ratio $D_{\mathrm{r}}=D_{\mathrm{H}} / D_{\mathrm{T}}$ varies over a large range, typically from $10^{-4}$ to $10^{1}$.

Figure 7 shows the change of the maximum pressure $P_{\max }$ and its location $x_{\mathrm{c}}$, with the diffusivity ratio $D_{\mathrm{r}}$. Table II gives typical values for two hydraulic conductivities. Depending on the diffusivity ratio, three ranges are obtained for $D_{\mathrm{r}}$. When $D_{\mathrm{r}}$ is less than $10^{-2}$, a high maximum pressure appears close to the hot face. Because a maximum tensile stress $\sigma_{1}^{\text {eff }}$ of nearly the same magnitude exists at the same location $(b=0.92)$, it may lead to the development of thin spalls parallel to the surface. The occurrence of damage depends on the material strength at these

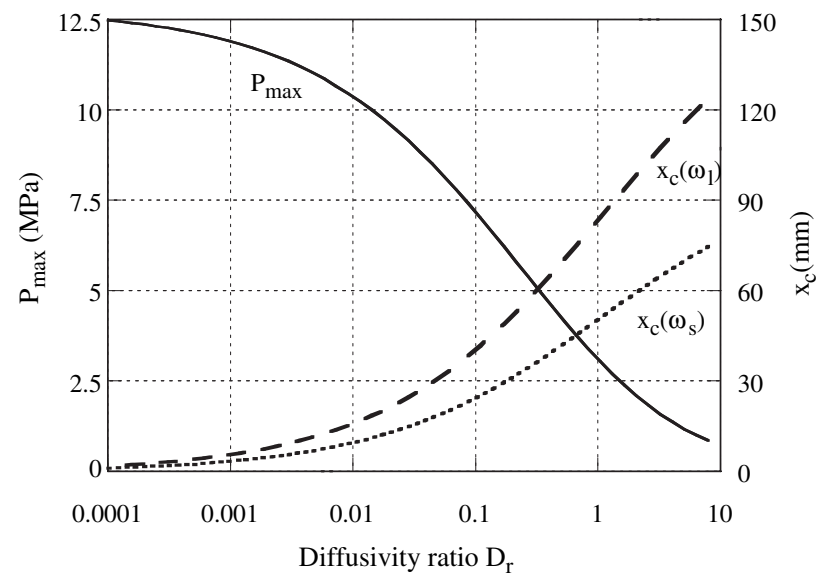

Fig. 7. Maximum pressure and location of the maximum pressure versus diffusivity ratio (longer cycle: $\omega_{1}=1.90 \times 10^{-4} \mathrm{rad} / \mathrm{s}$, shorter cycle: $\left.\omega_{\mathrm{s}}=5.24 \times 10^{-4} \mathrm{rad} / \mathrm{s}\right)$.
Table II. Typical Values of $\boldsymbol{P}_{\max }$ and $\boldsymbol{x}_{\mathrm{c}}$ for the Shorter Thermal Harmonic Cycle (Material Properties Defined for a Temperature of $1200^{\circ} \mathrm{C}$ )

\begin{tabular}{lccc}
\hline $\begin{array}{l}\text { Hydraulic } \\
\text { permeability } \\
k\left(\mathrm{~m}^{2} \mathrm{~Pa}^{-1} \cdot \mathrm{s}^{-1}\right)\end{array}$ & $\begin{array}{c}\text { Diffusivity } \\
\text { ratio } D_{\mathrm{r}}\end{array}$ & $\begin{array}{c}\text { Maximal interstitial } \\
\text { pressure } P_{\max }(\mathrm{MPa})\end{array}$ & $\begin{array}{c}\text { Critical abscissa } \\
x_{\mathrm{c}}(\mathrm{mm})\end{array}$ \\
\hline $10^{-12}$ & $8.4 \times 10^{-3}$ & 10.5 & 9 \\
$10^{-10}$ & $8.4 \times 10^{-1}$ & 3.4 & 48 \\
\hline
\end{tabular}

high temperatures. The value of $P_{\max }$ is close to the undrained interstitial liquid pressure $P_{\text {nd }}$ (Eq. (6)). Conversely, for $D_{\mathrm{r}}$ greater than 10 , the maximum pressure and the effective stress $\sigma_{1}^{\text {eff }}$ are low. They are located at depths greater than the impregnated zone of the lining. Consequently, the slag pressure developed by a harmonic thermal loading does not cause the degradation observed in refractory linings. In the intermediate range, a larger critical abscissa with a pressure less than $P_{\text {nd }}$ is likely to initiate larger spalls. This is precisely the domain of $D_{\mathrm{r}}$ covered by experiments.

Let us examine the stress levels in the zone where cracks are observed for the longer thermal cycle and an intermediate diffusivity ratio $D_{\mathrm{r}}=0.01$. During heating, at the location $x_{\mathrm{c}}$, $P_{\max }=10.5 \mathrm{MPa}, \sigma_{2}^{\text {eff }}=-9.6 \mathrm{MPa}$, and $\sigma_{2}^{\text {eff }}=-5.7 \mathrm{MPa}$, respectively. The existence of compressive stresses perpendicular to cracks at the origin of spalling increases the risk of their opening by buckling. However, these stresses are less than the pure thermal stresses (i.e., $-8.1 \mathrm{MPa}$ ) because the pressure of slag unloads the stresses applied to the skeleton by the thermal expansion. Although a tensile stress $\sigma_{2}^{\text {eff }}$ appears during the cooling phase of the thermal cycle with a maximum magnitude of 5.7 $\mathrm{MPa}$ (less than the maximum $\sigma_{1}^{\text {eff }}$ during heating), it does not generate cracks perpendicular to the free surface.

The previous remarks must be conducted with caution. First, let us note that the undrained pressure $P_{\mathrm{nd}}$ is a linear function of Young's modulus of the refractory skeleton (here $E=5 \mathrm{GPa}$ at $1200^{\circ} \mathrm{C}$ ). This property varies considerably with temperature (e.g., $E=1 \mathrm{GPa}$ when $T=1400^{\circ} \mathrm{C}$ ), so that the maximum pressure of the liquid slag and the thermal stresses are approximately 5 times less if $E=1 \mathrm{GPa}$.

The results are only valid for refractories having low viscosities or a yield stress of several MPa, so that the hypothesis of the thermoelasticity of the skeleton remains valid. For refractories with a high creep behavior, ${ }^{26}$ the "slag pressure" is notably lower because of stress relaxation. ${ }^{27}$ The overall tendencies observed in the present analysis are still identical. Even though the maximum pressure is overestimated, it provides a tool for checking the influence of material parameters on the onset of slag pressure and the stresses under periodic thermal histories.

Last, the harmonic thermal loading used herein is only a rough approximation for the usual loading. In particular, during the filling stage the temperature increases much faster than for a harmonic loading. The cooling stage is also faster during the first minutes, but the thermal shock is lower than for the heating stage.

\section{Summary}

A multi-physical framework is proposed to study the degradation of refractories impregnated by molten slag. Three major phenomena exist, namely, slag impregnation, thermomechanics, and phase transformations. A part of the interactions is used to investigate the onset of spalling in bauxite-based working lining of steel ladles due to slag impregnation, pressure fluctuations, and thermal stresses.

For the in-service temperature range, a liquid phase penetrates into the porosity of the bauxite brick and saturates a zone of several centimeters. The chemical composition of this phase differs from that of slag and varies with depth. The viscosity of the liquid depends on both the chemical composition and the 
temperature. Urbain's model revealed that it is much more sensitive to temperature than composition.

The presence of a fluid phase impregnating a refractory coupled with the cyclic thermal history generates a fluctuation of the interstitial pressure. A model is developed for quasi-brittle-impregnated refractories, which gives a closed-form solution for the maximum pressure in use, its location, and the thermal stresses. The main impact of the diffusivity ratio, the frequency, and amplitude of the thermal history on the pressure amplitude and its maximum location is shown. Even though a low hydraulic permeability could be interesting to limit impregnation, if the refractory is impregnated, it is harmful because a high pressure develops.

\section{Appendix A}

This appendix summarizes a simplified 1D analysis to obtain Eq. (2). The full calculations, starting from the linearized equation of isotropic thermoporoelasticity, ${ }^{21}$ are given in reference Blond et al. ${ }^{22}$ The porous medium is assumed to be made of a continuum skeleton filled by a continuum fluid phase. The momentum balance reads

$$
\frac{\partial \sigma}{\partial x}=0
$$

By considering Darcy's law and Fourier's law, the equations for fluid mass balance are written as

$$
\begin{aligned}
& k \frac{\partial^{2} P}{\partial x^{2}}=\frac{1}{\rho_{\mathrm{f} l}} \frac{\partial m}{\partial t} \\
& \frac{\partial^{2} \theta}{\partial x^{2}}=D_{\mathrm{T}} \frac{\partial \theta}{\partial t}
\end{aligned}
$$

where $\theta$ is the temperature and $D_{\mathrm{T}}$ the thermal diffusivity of the whole medium. The skeleton is assumed to be homogeneous, isotropic, and thermoelastic. Thus, the constitutive equation is given by

$$
\sigma=(\lambda+2 \mu) \varepsilon-b P-3 \alpha K \theta
$$

where $\lambda$ and $\mu$ are the Lamé coefficients, $\alpha$ the coefficient of thermal expansion, and $K$ the compressibility. The constitutive equation for the fluid is

$$
\frac{m}{\rho}=\frac{P}{M}+b \varepsilon-3 \alpha_{\mathrm{m}} \theta
$$

where $M$ is Biot's modulus, linked to porosity and skeleton and fluid compressibility. $b$ is Biot's coefficient, which is close to unity in the present application. $\varepsilon$ is the skeleton strain, $\alpha_{\mathrm{m}}$ a homogenized coefficient of thermal expansion dependent on porosity, Biot's coefficient, skeleton and fluid thermal expansion. Using the constitutive equation for the skeleton (A-4) in the momentum balance Eq. (A-1) gives one-dimensional Navier's equation. Its integration over $x$ for a problem involving a traction-free surface gives

$$
(\lambda+2 \mu) \varepsilon=b P+3 \alpha K \theta
$$

Similarly, by using the constitutive Eq. (A-5), the fluid mass balance $(b)$, and substituting the strain $\varepsilon$ by its expression

$$
\begin{array}{r}
\frac{\partial^{2} P}{\partial x^{2}}-\frac{1}{k}\left(\frac{1}{M}+\frac{b^{2}}{\lambda+2 \mu}\right) \frac{\partial P}{\partial t} \\
=\frac{3}{k}\left(\frac{K b}{\lambda+2 \mu} \alpha-\alpha_{\mathrm{m}}\right) \frac{\partial \theta}{\partial t}
\end{array}
$$

By using the heat diffusion Eq. (A-3), one obtains Eq. (2) and the definitions of hydraulic diffusivity and relative bulk variation

$$
\begin{aligned}
& D_{\mathrm{H}}=k\left(\frac{1}{M}+\frac{b^{2}}{\lambda+2 \mu}\right)^{-1} \\
& \delta_{v}=3\left(\frac{K b}{\lambda+2 \mu} \alpha-\alpha_{\mathrm{m}}\right)
\end{aligned}
$$

\section{Acknowledgments}

The authors wish to thank Dr. O. Arnould for his help in performing the microanalyses. This work was funded by an Arcelor grant.

\section{References}

${ }^{1}$ W. E. Lee and R. E. Moore, "Evolution of In Situ Refractories in the 20th Century," J. Am. Ceram. Soc., 81 [6] 1385-410 (1998).

${ }^{2}$ N. Bannenberg, "Demands on Refractory Material for Clean Steel Production"; pp. 19-22 in Proceedings of the Unified International Technical Conference on Refractories (UNITECR)' 95, TARJ, Kyoto, Japan, 1995.

${ }^{3}$ P. Blumenfeld, M. Puillet, J. de Lorgeril, and D. Verrelle, "Effect of Service Conditions on Wear Mechanisms of Steel Ladle Refractories"; in Proceedings of the Unified International Technical Conference on Refractories (UNITECR)' 97, American Ceramic Society, New Orleans, 1997.

${ }^{4}$ W. S. Chang, C. Knight, D. Hasselman, and R. Mitchiner, "Analysis of Thermal Stress Failure of Segmented Thick-Walled Refractory Structures," J. Am. Ceram. Soc., 66 [10] 708-13 (1983).

${ }^{5} \mathrm{~K}$. Andreev and H. Harmut, "Modelling of the Thermo-Mechanical Behavior of the Lining Materials of Teeming Ladles"; pp. 830-41 in Proceedings of the Unified International Technical Conference on Refractories (UNITECR)' 01, ALAFAR, Cancun, Mexico, 2001.

${ }^{6}$ W. D. Kingery, "Factors Affecting Thermal Stress Resistance of Ceramic Materials," J. Am. Ceram. Soc., 38 [1] 3-15 (1955).

${ }^{7}$ D. P. H. Hasselman, "Thermal Stress Resistance Parameters for Brittle Refractory Ceramics: A Compendium," Bull. Am. Ceram. Soc., 49 [12] 1033-7 (1970).

${ }^{8}$ N. Schmitt, Y. Berthaud, J. F. Hernandez, P. Meunier, and J. Poirier, "Damage of Monolithic Refractory Linings in Steel Ladles During Drying," Br. Ceram. Trans., 103 [3] 121-33 (2004)

${ }^{9}$ F. Quafssaoui, "Rôle de l'effet de l'andalousite sur le comportement à la corrosion des céramiques réfractaires à haute teneur en alumina"; $\mathrm{Ph}$. D. Thesis, in French, University of Orléans, France, 2004.

${ }^{10}$ F. Quafssaoui, J. Poirier, J. P. Ildefonse, and J. Hubert, "Influence of the Liquid Phase on the Slag Corrosion of Andalusite-Based Refractories," Refract. Appl. Trans., 1 [1] 2-8 (2005).

${ }^{11}$ S. E. McCune, T. P. Greaney, W. C. Allen, and R. B. Snow, "Reaction between $\mathrm{K}_{2} \mathrm{O}$ and $\mathrm{Al}_{2} \mathrm{O}_{3}-\mathrm{SiO}_{2}$ Refractories As Related to Blast-Furnace Linings," Am. Ceram. Soc., 40 [6] 187-95 (1957).

${ }^{12}$ S. Yabe, Y. Morita, T. Suzuki, K. Harada, and Y. Murakami, "Brittle Layer on Carbon Refractory in the Heart of A Blast Furnace," Interceram. Special Issue on Refractories, 32, 93-6 (1983).

${ }^{13}$ P. T. Josnes, B. Blanpain, P. Wollants, R. Dings, and B. Halemans, "Degradation Mechanisms of Magnesia-Chromite Refractories in Vacuum-OxygenDecarburisation Steel Ladles During Production of Stainless Steel," Iron. Steelmak., 27 [3] 228-37 (2000).

${ }^{14}$ E. Blond, "Dégradation thermomécanique des réfractaires au contact de laitiers sidérurgiques," Ph.D. Thesis, in French, ENS de Cachan, France, 2003.

${ }^{15} \mathrm{M}$. Onillon and J. Perrin, "Hydrodynamique et physico-chimie de l'attaque des réfractaires industriels par les laitiers sidérurgiques. Part I \& II," Bull. Soc. Française de Céram., 3 [12] 128-9 (1980).

${ }^{16} \mathrm{D}$. Xie and T. Tran, "Studies of Dynamic Refractory-Slag Interactions Using In-Situ Gravimetric Technique"; pp. 1418-31 in Proceedings of the Unified International Technical Conference on Refractories (UNITECR)' 01, ALAFAR Cancun, Mexico, 2001.

${ }^{17}$ J. Lehmann, M. Boher, H. Soulard, and C. Gatellier, "Metal/Refractory Interactions: A Thermodynamic Approach"; pp. 23-36 in Proceedings of the Unified International Technical Conference on Refractories (UNITECR)' 01, ALAFAR Cancun, Mexico, 2001.

${ }^{18}$ S. Zhang, H. R. Rezaie, H. Sarpoolkay, and W. E. Lee, “Alumina Dissolution into Silicate Slag,” J. Am. Ceram. Soc., 83 [4] 897-903 (2000).

${ }^{19}$ E. Levin, C. Robbins, and H. McMurdie, Phase Diagrams for Ceramists, pp. 142-32. American Ceramic Society, Columbus, OH 1964 (figs. 364-667).

${ }^{20}$ G. Urbain, "Viscosity Estimation of Slag," Steel Research, 58 [3] 111-6 (1987).

${ }^{21}$ O. Coussy, Mechanics of Porous Continua. J. Wiley and Sons Ltd, Chichester, 1995.

${ }^{22}$ E. Blond, N. Schmitt, and F. Hild, "Response of Saturated Porous Media to Cyclic Thermal Loadings," Int. Jal. Analyt. Num. Meth. Geomech., 27 [11] 883-904 (2003).

${ }^{23}$ D. F. McTigue, "Thermoelastic Response of Fluid-Saturated Porous Rock," J. Geophys. Res., B9 [91] 9533-42 (1986).

${ }^{24}$ I. Evenepoel, E. Piccaret, and P. Blumenfeld "Influence du réfractaire de poche sur la thermique du bain d'acier" (Influence of steel ladle refractory lining on the temperature of steel bath), CRDM Dunkerque/Arcelor report no. 9907-097R, 1999 (in French). 
${ }^{25}$ R. W. Vervoort and S. R. Cattle, "Linking Hydraulic Conductivity and Tortuosity Parameters to Pore Space Geometry and Pore-Size Distribution," J. Hy drol., 272, 36-49 (2003).

${ }^{26}$ E. Blond, N. Schmitt, F. Hild, P. Blumenfeld, and J. Poirier, "Modeling of High Temperature Asymmetric Creep Behavior of Ceramics," J. Eur. Ceram. Soc., 25 [11] 1819-27 (2005)
${ }^{27}$ N. Schmitt, F. Hild, and E. Blond, "Thermal Stresses in the Working Lining of a Ladle During the Steel Refining"; pp. 39-51 in Proceedings of the 4TH International Symposium on Advances in Refractories for the Metallurgical Industries, Edited by M. Rigaud and C. Allaire. Metsoc, Hamilton, Canada, 2004.

${ }^{28}$ Slag Atlas, 2nd edition, Verein Deutscher Eisenhuttenleute (VDEh), Verlag Stahleisen GmbH, Dusseldorf, ISBN 3-514-0457-9, 1995. 